\title{
Evaluation of Serum Neurokinin A Level in Alopecia Areata Patients
}

\author{
Abd El Aziz E. El-Taweel ${ }^{\text {a }}$, Essam El-Sayed Akl ${ }^{\text {a }}$, Asmaa A. El Fallah ${ }^{\text {b }}$, Marian N. William ${ }^{\text {a }}$
}

\footnotetext{
a Department of Dermatology, Venerology and Andrology, b department of Clinical and Chemical Pathology, Faculty of Medicine, Benha University, Egypt

Correspondence to: Marian N. Williamm department of Dermatology, Venerology and Andrology department, Faculty of medicine, Benha university, Egypt

email:

maro_medica@yahoo.com
}

Received: 7 July 2019

Accepted: 23 December 2019

BMJ 2019, vol 36, issue 2

\section{Background}

Alopecia areata (AA) is a complex autoimmune condition with increase of some pro-inflammatory mediators. The association of the novel neurokinin A with AA is still unclear. Data concerning other inflammatory diseases suggest a possible role of this neurokinin A in the pathophysiology of AA

\begin{abstract}
Aim
The aim of this work was to evaluate serum level of neurokinin A in patients with AA and the impact of the AA on dermatological life quality index (DLQI) and depression anxiety stress scale (DASS).
\end{abstract}

\section{Patients and Methods}

Neurokinin A concentrations were measured in 50 patients with AA and 30 healthy controls using enzyme-linked immunosorbent assay (ELISA).

\section{Results}

The mean serum levels of neurokinin A was higher in patients than controls with significance $\mathrm{p}$ value is $(<0.001)$. It was higher in male patients than females with no significance $(p=0.668)$. It was statistically significant higher in patient with chronic disease $(\mathrm{p}=0.023)$. There was statistically significant positive correlation found between serum neurokinin A level and severity of alopecia tool (SALT) score and stress in DASS subscales (as, $\mathrm{p}=<0.001, \mathrm{p}=0.025$ ) respectively

\section{Conclusion}

The mean serum levels of neurokinin A was higher in patients than controls with significance $\mathrm{p}$ value. It was statistically significant higher in patient with chronic disease also There was statistically significant positive correlation between serum neurokinin A level and SALT score and stress in DASS subscales.

Key words: Alopecia areata; neurokinin A; substance p. 


\section{Introduction}

Alopecia areata (AA) is a disorder characterized by transient, non-scarring hair loss and preservation of the hair follicle, with prevalence approximately $2 \%$ of the general population at some point during their lifetime (1). Spontaneous regrowth of hair occurs in $80 \%$ of patients within the first year, but relapses at any given time are not uncommon (2).

Neurokinin A is ubiquitous in both the central and peripheral mammalian nervous systems, and seems to be involved in reactions to pain and the inflammatory responses (3). Substance P (SP) and neurokinin A (NKA) are expressed by the same gene (4). The

\section{Subjects and methods}

This comparative case control study included 50 patients with AA and 30 healthy, age and sex matched controls attending the dermatology outpatient clinic at Benha University Hospital, Benha, Egypt from July 2018 to March 2019. Participants gave their informed written consent before enrolment and the study was approved by the Research Ethics Committee in Faculty of Medicine, Benha University.

All patients included in the study, were with AA variable size, duration less than one year, all patients included from both sexes between the age of 16 and 35 years. Patients with other causes of hair loss, kidney or liver diseases, other autoimmune diseases and other psychiatric disorders and any subject who is currently experiencing significant spontaneous regrowth of terminal hair were excluded. expression of the neuropeptide SP and its effects on immune cells in a mouse model for alopecia areata have been examined and the number of SP immunoreactive nerve fibers in skin was found to be increased in evolving alopecia areata (5).

No data exist on neurokinin $\mathrm{A}$ in the context of AA. However, data from other inflammatory diseases suggest a possible role of this Neurokinin A in AA.

The aim of this study was to evaluate serum level of NKA in patients with alopecia areata and the impact of the AA on DLQI and DASS.

None of the patients was on systemic or topical treatment for one month before the study.

Disease severity was assessed Severity of Alopecia Tool score (SALT score) (6). AA was diagnosed clinically.

Dermatology life Quality index (DLQI) (7) was assessed using the validated Arabic version of the 10-item Dermatology Life Quality Index (8) to determine the effect of AA on patients' quality of life (QOL).

Depression, Anxiety and Stress Scale (DASS21) (9) was self-assessed by participants using an Arabic version of the 21-item to screen symptoms of Depression, Anxiety and Stress (10).

\section{Laboratory investigations}

Five milliliters of venous blood were withdrawn from each patient and control subjects, the blood samples were allowed to clot completely (within 30minutes) at the room temperature, centrifugation 
was done at $3000 \mathrm{rpm}$ for 10 minutes to separate the serum. After centrifugation, serum was separated by a pipette, divided and kept in two eppendorf tubes labeled with the number of the person. Specimens were kept at

\section{Statistical methods:}

Statistical analysis was performed using data management and statistical analysis was done using SPSS vs.25.

\section{Results:}

This study included 80 subjects, 50 AA patients and 30 normal subjects as a control. No statistically significant differences were found between patients and controls regarding age and sex(Table 1).

The mean duration of disease in patients group was $14.1 \pm 26.9$ months, and the mean SALT score was $(27.9 \pm 23.8)$

High DQLI scores were reported among patients group indicating quality of life impairment among AA patients, most common score was very large $31(62 \%)$ followed by moderate in $15(30 \%)$ $-20^{\circ} \mathrm{C}$ to preserve until the time of the run. Serum neurokinin A levels were determined using Human NKA (Neurokinin A) ELISA kit provided by Elabscience® / USA then extremely large in 3(6\%) and small in $1(2 \%)$ while there was no effect in DQLI score among control group, there was statistically significant difference between patients and control regarding DLQI as $p$ value $(<0.001)$ (table 2$)$

The most apparent negative emotion among AA patients was moderate depression 22 cases $(44 \%)$ followed by extremely severe stress 18 cases (36\%), there was statistically significant difference between patients and control regarding depression, anxiety and stress as regard all of them $\mathrm{P}$ value $(<0.001)$ (table 3)

Table (1) Demographic and Descriptive data of patients and controls

\begin{tabular}{llll}
\hline Variables & $\begin{array}{l}\text { Patients } \\
(\mathbf{n}=50)\end{array}$ & $\begin{array}{c}\text { Controls } \\
(\mathbf{n}=30)\end{array}$ & P value \\
\hline
\end{tabular}

Age Mean $\pm \mathrm{SD}$ (years)

$29 \pm 6$

$30 \pm 6$

0.605

Sex

Male $n(\%)$

Female $n(\%)$

$27(54 \%)$

$16(53.3 \%)$

Duration of disease (Mean \pm SD)

(Months)

$23(46 \%)$

$14(46.7 \%)$

$14.1 \pm 26.9$

SALT score (Mean \pm SD)

$27.9 \pm 23.8$

Recurrence $n(\%)$

Yes

$$
n(\%)
$$

$16(32.0)$ 
Table (2): comparison between patients and controls regarding DLQI score

\begin{tabular}{lccc}
\hline DLQI score & Patients(n=60) \% & Controls(n=30) \% & P value \\
\hline No effect & $0(0 \%)$ & $30(100)$ & $<0.001 *$ \\
Small & $1(2 \%)$ & $0(0 \%)$ & \\
Moderate & $15(30 \%))$ & $0(0 \%)$ & \\
Very large & $31(62 \%)$ & $0(0 \%)$ & \\
Extremely large & $3(6 \%)$ & $0(0 \%)$ & \\
\hline
\end{tabular}

Table (3) Comparison between patients \& controls regarding DASS score

\begin{tabular}{|c|c|c|c|c|}
\hline $\begin{array}{l}\text { DASS } \\
\text { subscale }\end{array}$ & Score & Patients $(n=60) \%$ & Controls $(\mathrm{n}=\mathbf{3 0}) \%$ & $p$ value \\
\hline \multirow[t]{5}{*}{ Depression } & Normal & $10(20 \%)$ & $25(83.3 \%)$ & $<0.001 *$ \\
\hline & Mild & $8(16 \%)$ & $3(10 \%)$ & \\
\hline & Moderate & $22(44 \%)$ & $2(6.6 \%)$ & \\
\hline & Severe & $3(6 \%)$ & $0(0 \%)$ & \\
\hline & Extremely severe & $7(14 \%)$ & $0(0 \%)$ & \\
\hline \multirow[t]{5}{*}{ Anxiety } & Normal & $6(12 \%)$ & $28(93.3 \%)$ & \\
\hline & Mild & $10(20 \%)$ & $1(3.3 \%)$ & \\
\hline & Moderate & $16(32 \%)$ & $1(3.3 \%)$ & \\
\hline & Severe & $11(222 \%)$ & $0(0 \%)$ & \\
\hline & Extremely severe & $7(14 \%)$ & $0(0 \%)$ & \\
\hline \multirow[t]{5}{*}{ Stress } & Normal & $2(4 \%)$ & $27(90 \%)$ & \\
\hline & Mild & $7(14 \%)$ & $2(6.6 \%)$ & \\
\hline & Moderate & $16(32 \%)$ & $1(3.3 \%)$ & \\
\hline & Severe & $18(36 \%)$ & $0(0 \%)$ & \\
\hline & Extremely severe & $7(14 \%)$ & $0(0 \%)$ & \\
\hline
\end{tabular}

mean serum neurokinin A level was statistically highly significant in controls than patients with a $\mathrm{p}$ value $(p<0.001)($ table 4). In patient group; neurokinin A level was statistically non-significant $\mathrm{p}$ value $(\boldsymbol{p}=0.668)$ (table 5). Neurokinin A 
level was statistically significant higher in patient with chronic disease $(\mathrm{p}=0.023)$ (table 6). There was no statistically significant correlation found between serum serum Neurokinin A level and Age (years), duration ( month ), DLQI, Depression and Anxiety (table 7).

Table (4): mean serum neurokinin A level between patients and controls

\begin{tabular}{llll} 
& Patients $(\mathbf{n}=\mathbf{5 0})$ & Controls $(\mathbf{n}=\mathbf{3 0})$ & p-value \\
\hline $\begin{array}{l}\text { Neurokinin A (Mean } \pm \text { S.D) } \\
(\mathrm{ng} / \mathrm{ml})\end{array}$ & $94.2 \pm 38.9$ & $64.6 \pm 23.4$ & $<0.001$ \\
\hline
\end{tabular}

Table (5): Mean serum levels of neurokinin A level both sexes in patients

\begin{tabular}{llll} 
& Males & Females & p-value \\
\hline $\begin{array}{l}\text { Neurokinin A (Mean } \pm \text { S.D) } \\
(\mathrm{ng} / \mathrm{ml})\end{array}$ & $95.4 \pm 37.7$ & & \\
\hline
\end{tabular}

Table 6): Mean neurokinin A levels in patients with different disease durations

\begin{tabular}{llll} 
& $\leq 1$ year & $>$ 1 year & p-value \\
\hline $\begin{array}{l}\text { Neurokinin A (Mean } \pm \text { S.D) } \\
(\mathrm{ng} / \mathrm{ml})\end{array}$ & $85 \pm 32$ & $115.6 \pm 45.9$ & 0.023 \\
\hline
\end{tabular}

Table (7): Correlation between Neurokinin A and different variables in patients

\begin{tabular}{lcc}
\hline $\begin{array}{l}\text { Variable } \\
\text { (No.=50) }\end{array}$ & $\begin{array}{c}\text { Neurokinin A } \\
(\mathbf{n g} / \mathbf{m l})\end{array}$ & p-value \\
\hline Age (years) & $\mathbf{r}^{*}$ & 0.277 \\
SALT score & -0.175 & $<0.001$ \\
DQLI & $0.593^{* *}$ & 0.906 \\
DASS subscales & 0.017 & \\
Depression & & 0.936 \\
Anxiety & -0.012 & 0.336 \\
Stress & -0.139 & 0.025 \\
\hline
\end{tabular}

\section{Discussion}

Several hypothesis have been proposed in AA pathogenesis including autoimmune, vascular, hormonal and genetic processes, a diet deficient in iron (11), mental factors and disorders in nervous system are subjected to have role in alopecia areata .Researches show that the autoimmune background seems to be the most probable factor of AA, 
Around hair follicle, $\mathrm{T}$ lymphocytes accumulate (predominantly Th1) and macrophages which release cytokines and exacerbate inflammation that may have a role in alopecia areata (12).

Release of substance $\mathrm{P}$ from peripheral nerves in response to stress has also been reported, and prominent substance $\mathrm{P}$ expression is observed in nerves surrounding hair follicles in alopecia areata patients (13). Substance P-degrading enzyme neutral endopeptidase has also been strongly expressed in affected hair follicles in the acute-progressive as well as the chronic stable phase of the Alopecia areata. Comorbid psychiatric disorders are also common among AA patient and include major depression, generalized anxiety disorder, phobic states, and paranoid disorder (14).

The aim of this work was to evaluate serum level of neurokinin A in patients with alopecia areata and the impact of the AA on (DLQI) and DASS.

To achieve this goal, this study was conducted on 80 subjects; 30 normal subjects and 50 patients with AA

The mean age of patients in the study was (29 \pm 6 ) years (ranged from 16 - 35 years), while the mean age of controls was $(30 \pm 6)$ years.

Kyriakis et al .2009 showed that the median age for men was 30 years and 31 years for women. Overall both sexes were equally affected and peak prevalence was observed in the age group of 31-40 years. (15)

Patients group included 27 (54\%) males and $23(46 \%)$ females, while controls included 16 (53.3\%) males and 14 (46.7\%) females. There was no statistically significant difference regarding gender. That was matched with Mirzoyev et al.,2014 study in which number of significant difference in the incidence of AA was found between males and females in either of the two population studies (16) On the other hand, Lundin et al., 2014 showed that, a higher incidence rate of AA in the female population is also described. Male AA patients are more likely to be diagnosed in childhood (age $<10$ years) and have a family history of AA. On the other hand, female AA patients are more likely to be diagnosed in adolescence (age 10-20 years), have co-morbid nail involvement, and have concomitant autoimmune disease, particularly thyroid disease (17).

In two epidemiological studies by Lazzarini et al .,2016 men under 50 years of age were more affected, while women over 50 were slightly more affected (18).

The mean duration of AA in patient group was $14.1 \pm 26.9$ months 35 cases $(70 \%)$ were less than 1 year duration and 15 cases $(30 \%)$ were with more than 1 year duration and the mean of recurrence rate was 16 (32.0).

High DQLI scores was reported among patients group indicating quality of life impairment among AA patients, most common score was very large in 40 (66.7\%) followed by moderate in $14(23 \%)$ then extremely large in $4(6.7 \%)$ and small in $2(3.3 \%)$. There was statistically significant difference between patients and control regarding DLQI.

This result is matched with study by $\boldsymbol{A l}$ Mutairi and Eldin in 2011, who observed 2962 patients with AA including 1926 
males and 1036 females patients. Women exhibited a $13.6 \%$ incidence of severe disease with marked disturbances in their social life, which forced them to miss school or social meetings, change their hairstyles, or alter their type of clothing (19).

There is also another study by $\boldsymbol{Q i}$ et $\boldsymbol{a l}$ in 2015, using the Chinese version of DLQI questionnaire. In their study patients with alopecia totalis / alopecia universalis, longer duration and recurrent disease exhibited higher scores. Patients with AA treated early, and requires psychological support in addition to prescription drugs (20). The study used the DASS-21 to assess impact of stress on AA. Results reported significant difference in depression, anxiety and stress between AA patients and control, the most apparent negative emotion among AA patients was moderate depression 22(44\%) followed by severe stress $18(36 \%)$.

Alshahwan et al .2015 used DASS in skin diseases including psoriasis, hair loss and other skin diseases and found that the frequency of anxiety and depression in Arab dermatology patients was $29 \%$ for anxiety and $14 \%$ for depression and patients suffering from hair loss had the highest anxiety and depression scores. (21).

Results of the present study showed that there is significant elevation of neurokinin $\mathrm{A}$ in AA patient than control. In addition serum neurokinin $\mathrm{A}$ is elevated in patient with more disease severity.

In patients with less than 1 year duration mean Neurokinin A level was $(85 \pm 32)$ $\mathrm{ng} / \mathrm{ml}$ and with more than 1 year was $(115.6 \pm 45.9) \mathrm{ng} / \mathrm{ml}$.
In a study done by Amatya ( 2011 ) there were increase in the number of substance P- and NKA-immunoreactive fibers and cells in lesional compared with nonlesional psoriatic and healthy control skin. This indicates a possible role of NKA as a pruritogenic mediator in psoriasis (22).

Substance $\mathrm{P}$ and NKA are expressed by the same gene. [4] .In accordance with the existence of a common precursor for SP and NKA, NKA is known to coexist with $\mathrm{SP}$ in sensory neurons in a large number of tissues (23).

Psychological/psychiatric disturbances also contribute to the complex puzzle of etiologic theories for AA. These include the presence of a stressful life event as a trigger of the outbreak, the presence of comorbidity between psychiatric disorders and AA, and the patient's personality (24).

Neurokinin A is also detected in other hypersensitivity diseases such as bronchial asthma and was found that NKA have potent effects on bronchomotor tone, airway secretions and bronchial circulation. Sputum NKA is up-regulated during acute asthma exacerbation and it positively correlates to its severity (25).

Stress has been reported to play a role in the onset and exacerbation of psoriasis, which might include the substance P-NK-1 receptor (R) pathway. A release of neuropeptides from cutaneous nerve fibers in response to stress has been suggested. The substance P-neurokinin (NK)-1 receptor $(\mathrm{R})$ pathway has been shown to be activated in response to stressful (26).

The expression of the neuropeptide substance P (SP) and its effects on immune cells in a mouse model for alopecia areata have been examined, and the number of SP 
immunoreactive nerve fibers in skin was found to be increased in evolving alopecia areata. In contrast, in more advanced stages of the disease, the number of SP immunoreactive nerves and SP protein levels in skin were found to be decreased, due to the SP degrading enzyme neutral endopeptidase increased. These results suggest that SP, neutral endopeptidase, and NK-1R can serve as regulators in the molecular signaling network modulating pathogenesis of AA by other researchers (5).

\section{Conclusion;}

In this study there was a significant increase of the mean serum level of neurokinin A in AA patients compared with controls.there was positive correlation between severity and serum neurokinin A level with statistically significant value. Regarding DASS, there was statistically significant difference between patients and control regarding depression, anxiety and stress Alopecia areata also cause a negative impact on patient quality of life as there was statistically significant difference between patients and control regarding DLQI .

\section{Reference}

1 Pratt $\mathrm{CH}$, King LE Jr, Messenger AG, Christiano .AM, Sundberg JP

(2017): Alopecia areata .Nat Rev Dis Primers. $16 ; 3: 17011$

2 Roest YBM, van Middendorp HT, Evers AWM, van de Kerkhof PCM, Pasch MC (2018): Nail Involvement in Alopecia Areata: A Questionnairebased Survey on Clinical Signs, Impact on Quality of Life and Review of the Literature . Acta Derm Venereol. 7;98(2):212-217 inflammatory response in autoimmune hair loss such as alopecia areata (5).

The expression of neuropeptides and neuropeptide receptors by nerves as well as immune cells provides a link between the peripheral nervous system, the central nervous system, and the skin immune system (27).

The result of this study is similar to previous study about other takikinin role in

\section{Financial support and sponsorship \\ Nil.}

\section{Conflicts of interest}

There are no conflicts of interest

3 Nakanishi S (2006) : Molecular mechanisms of intercellular communication in the hormonal and neural systems. IUBMB Life.; 58(5-6): 349-57

4 Krause JE, Chirgwin JM, Carter MS, Xu ZS, Hershey AD (1987) : Three rat preprotachykinin mRNAs encode the neuropeptides substance $\mathrm{P}$ and neurokinin A . Proc Natl Acad Sci U $S$ A.; 84(3):881-5

5 Siebenhaar F, Sharov AA, Peters EM, Sharova TY, Syska W, Mardaryev AN et al ., ( 2007) : Substance $\mathrm{P}$ as an immunomodulatory neuropeptide in a mouse model for autoimmune hair loss (alopecia areata). J Invest Dermatol.; 127(6):148997

6 Amin, S.S. and Sachdeva (2013):

Alopecia areata a review. Journal of the Saudi Society of Dermatology \& Dermatologic Surgery.; 17: $37-45$

7 Finlay AY and Khan GK. Dermatology life quality index (DLQI) — a simple practical measure 
for routine clinical use. Clin Exp Dermatol. 1994;19:210-6.

8 Khoudri ,I.; Lamchahab ,F.Z.; Ismaili ,N.;Senouci ,K.;Hassam, B.;Abouqal ,R. Measuring quality of life in patients with psoriasis using the Arabic version for Morocco of the Dermatology Life Quality IndexInt. JDermatol.2013;52(7):795802.

9 Lovibond, P.F and Lovibond ,S.H.

The structure of negative emotional states: compari son of the Depression Anxiety Stress Scales(DASS) with the Beck Depression and Anxiety Inventories. Behav Res Ther. 1995;33(3):335-43.

10 Taouk ,M.;Lovibond ,P.F.: Laube R. Psychometric properties of an Arabic version of the Depression Anxiety Stress Scales (DASS). Research on Social Work Practice. 2017;27(3) 37538

11 Wang E, McElwee KJ (2011) : Etiopathogenesis of alopecia areata: Why do our patients get it? . Dermatol Ther.;24(3):337-47

12 Kuty-Pachecka M.(2015): Psychological and psychopathological factors in alopecia areata. Psychiatr. Pol.; 49:955-964.

13 Toyoda M, Makino T, Kagoura M, Morohashi M ( 2001) : Expression of neuropeptide-degrading enzymes in alopecia areata: an immunohistochemical study. $\mathrm{Br} \mathrm{J}$

Dermatol.;144(1):46-54

14 García-Hernández MJ, Ruiz-Doblado S, Rodriguez-Pichardo A, Camacho F ( 1999) : Alopecia areata, stress and psychiatric disorders: a review. J Dermatol.;26(10):625-32

15 Kyriakis KP, Paltatzidou K, Kosma E, Sofouri E, Tadros A, Rachioti E ( 2009) : Alopecia areata prevalence by gender and age. J Eur Acad Dermatol Venereol.;23(5):572-3

16 Mirzoyev SA, Schrum AG, Davis MDP, Torgerson RR ( 2014) : Lifetime incidence risk of alopecia areata estimated at $2.1 \%$ by Rochester Epidemiology Project, 1990-2009. J Invest Dermatol.;134(4):1141-1142
17 Lundin M, Chawa S, Sachdev A, Bhanusali D, Seiffert-Sinha K, Sinha AA (2014): Gender differences in alopecia areata. J Drugs Dermatol.;13(4):409-413

18 Lazzarini R, Oliari CB, Erthal AL ( 2016) : Late-onset alopecia areata: descriptive analysis of 30 cases. An Bras Dermatol. ;91(6):844-845

19 Al-Mutairi N and Eldin ON (2011): Clinical profile and impact on quality of life: seven years' experience with patients of alopecia areata . Indian J Dermatol Venereol Leprol;77: 489_493

20 Qi S, Xu F, Sheng Y, Yang Q (2015): Assessing quality of life in Alopecia areata patients in China. Psychol health med.: 20 (1):97-102.

21 AlShahwan MA (2015): The Prevalence of Anxiety and Depression in Arab Dermatology Patients. J Cutan Med Surg; 19(3):297-303

22 Amatya B, El-Nour H, Holst M, Theodorsson E, Nordlind K (2011) : Expression of tachykinins and their receptors in plaque psoriasis with pruritus. Br J Dermatol.;164(5):1023-9

23 Dalsgaard CJ, Haegerstrand A, TheodorssonNorheim E, Brodin E, Hökfelt T (1985) : Neurokinin A-like immunoreactivity in rat primary sensory neurons; coexistence with substance P. Histochemistry.;83(1):37-9

24 Güleç AT, Tanriverdi N, Dürü C, Saray Y, Akçali C (2004) : The role of psychological factors in alopecia areata and the impact of the disease on the quality of life . Int J Dermatol. ;43(5):352-6

25 Mostafa GA, Reda SM, Abd El-Aziz MM, Ahmed SA. ( 2008) : Sputum neurokinin A in Egyptian asthmatic children and adolescents: relation to exacerbation severity. Allergy. 2008 Sep;63(9):1244-7

26 Remröd C, Lonne-Rahm S, Nordlind K (2007): Study of substance $\mathrm{P}$ and its receptor neurokinin-1 in psoriasis and their relation to chronic stress and pruritus . Arch Dermatol Res.;299(2):85-91

27 Hordinsky MK (2013): Overview of alopecia areata. J Investig Dermatol Symp Proc.;16(1):S135 .

To cite this article: Abd El Aziz E. El-Taweel , Essam El-Sayed Akl , Asmaa A. El Fallah , Marian N. William Evaluation of Serum Neurokinin A Level in Alopecia Areata Patients BMFJ 2019: 36 (2) DOI: 10.21608/bmfj.2019.14505.1010 